\title{
JUGANDO EN UN ESPACIO DE FANTASÍA. CLAVES PARA ELABORAR UNA JORNADA DE AVENTURA EN UN CENTRO DE ENSEÑANZA
}

\section{Autores}

\section{Resumen}

Pablo Caballero Blanco

David Sayago Zambrano

Guadalupe Dominguez Carrillo

Óscar Pérez Sáinz

Manual Parra Boyero
Facultad de Ciencias del Deporte Universidad de Extremadura

Para sentir y llegar al corazón de lo que una jornada de aventura es, tenemos que recordar el niño que un día fuimos y mirar con los mágicos ojos de la imaginación; sólo ellos nos permitirán entrar en un mundo de aventuras fantásticas donde todo es posible. ¿Te atreves?

Palabras clave: Aventura, experiencia, educación, juego y naturaleza

\section{Introducción}

«...la aventura presenta desarrollos de muy diversa naturaleza, perdiendo en ocasiones sus tradicionales límites de acción, adoptando esquemas de muy diversos planteamientos y dispar naturaleza. Son múltiples las actividades de aventura posibles en los centros, nos encontramos con un bloque en expansión, con unos horizontes totalmente imprevisibles de acción, magia e innovación curricular... las propuestas en cambio constante, implican un profesor o una profesora adaptado a los nuevos tiempos o necesidades, al día en el uso y disfrute de nuevos materiales y recursos tecnológicos. La aventura en cualquiera de sus facetas implica adentrarse en territorios de lo desconocido y eso cautiva inmediatamente al alumnado y al docente, aglutinando ante sí, diferentes logros y vivencias, generando espacios comunes de ocio y diversión.» (Parra y Rovira, 2002).

En el actual sistema educativo de Extremadura, aparece el bloque de Actividades Físico Deportivas en el Medio Natural (a partir de ahora AFIN), dentro de la etapa de secundaria. Este bloque se muestra como una fuente inagotable de posibilidades y de recursos (un gran espacio educativo), acorde con lo que seduce e inquieta a los alumnos. En ocasiones, debido a la falta de formación del pro- fesorado, a problemas organizativos, a la cultura escolar, a las características propias de estas actividades y al miedo-respeto que genera, sigue siendo una gran desconocida, y por lo tanto, está poco explotada.

Sin embargo, encontramos un nutrido grupo de autores, como Alonso (1992), Guillén y Lapetra (1995), Gutiérrez Sanmartín y González de Natos (1995), que ven dentro de las AFIN, un cúmulo de posibilidades de acción, con grandes atributos educativos, exportables a los centros de enseñanza. Por ejemplo, Gutiérrez Sanmartín y González de Natos (1995) afirman que los programas de aire libre y aventura, cada vez se están considerando con más intensidad en los programas educativos, incorporando numerosas actividades de aventura que pretenden desarrollar el carácter humano, la autoestima, la autoimagen, la autorrealización y las relaciones interpersonales.

Parra y col. (2000) hacen un recorrido amplio por los diferentes valores educativos que se encuentran en estas propuestas.

El motivo del presente artículo es poner en manos de los diferentes profesionales, las claves organizativas que les permitan la puesta en marcha 
de estas actividades, dentro de los centros de enseñanza. De tal manera, proponemos la realización de una jornada de aventura, que sirva como motor de nuevas actividades curriculares y como colofón de las existentes dentro del desarrollo del bloque de contenidos.

Para ello, vamos a dividir la organización del evento en cuatro tiempos organizativos. Los dos primeros hacen referencia a las decisiones previas a la actividad, el tercero a la puesta en marcha de la jornada y el cuarto a la reflexión y análisis de lo acontecido para mejorar el proyecto en años venideros.

\section{Realización de una jornada aventura en un centro de enseñanza}

Niños corriendo detrás de un contenedor, profesores mojados alrededor de un plástico, un barranco en las escaleras del instituto, descensos de paredes rocosas que parten de las ventanas, escalada para ciegos, redes colgantes que nacen desde la azotea del edificio, puentes monos entre los árboles, ... que difícil es explicar una jornada de aventura.

Sin duda es una actividad llena de dinamismo, donde todo el centro se ve involucrado en una gran aventura. En este día, conseguimos «darle la vuelta al centro», explotando todos los espacios clandestinos o no, con el objetivo de poder realizar las actividades que normalmente se desarrollan en el medio natural, generando propuestas que invitan a aprender, a probar, a indagar.

Son varios los autores que resaltan la posibilidad de traerse el medio natural al centro de enseñanza, adaptando las actividades o los espacios tradicionales de los mismos. Destacamos la opinión de Farias y Torrebadella (1995), quienes afirman que podemos imitar a la naturaleza confeccionando de forma artificial un espacio natural. El resultado se aproxima al concepto de espacio semiartificial, en el que se transforma o varía el estado inicial o natural del entorno de prácticas, pretendiendo reducir el nivel de riesgo objetivo y facilitando con ello la tarea.

Creemos que la práctica vivenciada proporciona (tanto al profesor/a como al alumno/a) experiencias, sensaciones, interrelaciones,... a través de los cuales podemos construir actitudes y hábitos, que favorezcan el crecimiento personal (proceso de humanización). Esta forma de trabajar se basa en la "pedagogía de la aventura» (Parra y col., 2002), siendo ésta una vertiente específica de la «pedagogía de la experiencia» de Ziegenspeck (1996).

\section{Objetivos}

Entre los objetivos y finalidades que se pueden lograr con esta propuesta, destacamos los siguientes:

a) Divertir y canalizar el ocio, favoreciendo los procesos de humanización.

En la actualidad, los jóvenes disponen de gran cantidad de tiempo de ocio, que disfrutan fundamentalmente los fines de semana, empleándolo casi en su totalidad en «la movida juvenil» (botellón, discotecas,...), planteando numerosos problemas sociales.

La observación de esta realidad, dirige indefectiblemente nuestra mirada hacia una concepción educativa del ocio, dentro de lo que autores como Cuenca (1995), denomina direccionalidad positiva del ocio; esto es, el impulso de propuestas y programas desde una dimensión del tiempo libre afín a metas como la humanización, el crecimiento personal y social, o las actitudes solidarias y tolerantes.

b) Buscar sensaciones.

Zuckerman (1979) citado por Gutiérrez San Martín y González de Martos (1995) describe este objetivo como «la necesidad por la variedad de sensaciones y experiencias nuevas y complejas, y la predisposición para asumir riesgos físicos y sociales por el puro placer de sentirlos».

Este autor, propone una escala de búsqueda de sensaciones que llevada al ámbito educativo, nos permite proporcionar al alumno/a actividades no usuales, que persiguen la búsqueda de emoción estimulando su pensamiento y sus sentidos.

Gracias a la práctica de este tipo de actividades se pueden experimentar una gran cantidad de sensaciones, entre las que podemos destacar: bienestar, placer, satisfacción, emoción, estimulación, excitación, sensación de libertad, miedo, nerviosismo, catarsis, independencia, autosuperación, euforia, relajación, aislamiento, vértigo y desafío. (Gutiérrez San Martín y González de Martos, 1995).

c) Sentir el riesgo.

Podemos clasificar el riesgo en los deportes de aventura con una doble vertiente: riesgo objetivo y subjetivo. Por un lado, el riesgo objetivo es aquel que está presente en las actividades y no se puede eliminar. El subjetivo hace referencia a la percepción que tiene la persona cuando realiza este tipo de prácticas.

En el caso de nuestras propuestas, tratamos de controlar en la medida de lo posible, el riesgo objetivo con los elementos de seguridad necesarios. 
Mientras que el riesgo subjetivo es un instrumento con el que tratamos de provocar diferentes sensaciones en el individuo, gracias a la creación de espacios de aventura.

d) Experimentar emociones.

Estas actividades nos sirven como medio de catarsis para evadirse de la vida cotidiana y conocerse más a uno mismo, sintiéndose bien y superándose.

Esto se logra haciendo que el sujeto sea en todo momento el protagonista activo de su historia, pasando de realizar el papel de simple espectador al de actor principal.

e) Buscar efectos positivos para la salud, el bienestar personal y grupal.

En relación a los efectos psicosociales, esta actividad provoca cambios en los comportamientos y actitudes de los sujetos, produciéndose una mejora de la autoestima, aumento de la autoconfianzay mayor conocimiento personal. Además fomentan actitudes de cooperación grupal, camaradería, pertenencia a un grupo, respeto al otro.

\section{Fases de la aventura organizativa}

A raíz de nuestras experiencias en la organización y puesta en práctica de jornadas de aventura en varios centros de enseñanza, hemos elaborado una «guía» que incluya todos los pasos a seguir para su realización.

\section{FASE PREACTIVA}

\section{PRIMER TIEMPO ORGANIZATIVO}

(Nace el proyecto, se persigue la viabilidad del mismo)

1. Realización de un anteproyecto:

- Sencillo y claro.

- Incluir imágenes (fácil de leer).

2. Preparación del claustro:

- Dar a conocer el proyecto.

- Incluirlo en el orden del día.

- Buscar apoyos entre compañeros.

3. Defensa en la Comisión de Coordinación Pedagógica y en el Claustro:

- Resaltar los aspectos educativos.

- Invitar al resto de áreas.

- Enfatizar en los aspectos organizativos, de control de la actividad y de la contingencia.
- Implicar al centro en un proyecto común (multidisciplinar).

4. Presentación del proyecto en el Consejo Escolar:

- Explicación del anteproyecto.

- Buscar la implicación de padres, personal no docente,...

- Fomentar la difusión del proyecto mediante los alumnos del consejo escolar.

5. Enrolar en la aventura a otros profesores:

Conseguir ilusionar a los compañeros afines y a los nuevos con la actividad.

- Neutralizar a los profesores más reacios a esta actividad.

- Ofrecer un «espacio» dentro del proyecto para cada profesor.

- Delegar responsabilidades e integrar sus aportaciones.

- Generar buen ambiente de trabajo en el centro (entre profesor-profesor, profesoralumno,...).

- Intentar que se reconozca la actividad como curso de formación permanente del profesorado (conseguir puntos'! motivaciones extrínsecas).

6. Incorporar a los alumnos en el proyecto: Acoger a determinados alumnos para colaborar en la organización y hacer el proyecto más suyo.

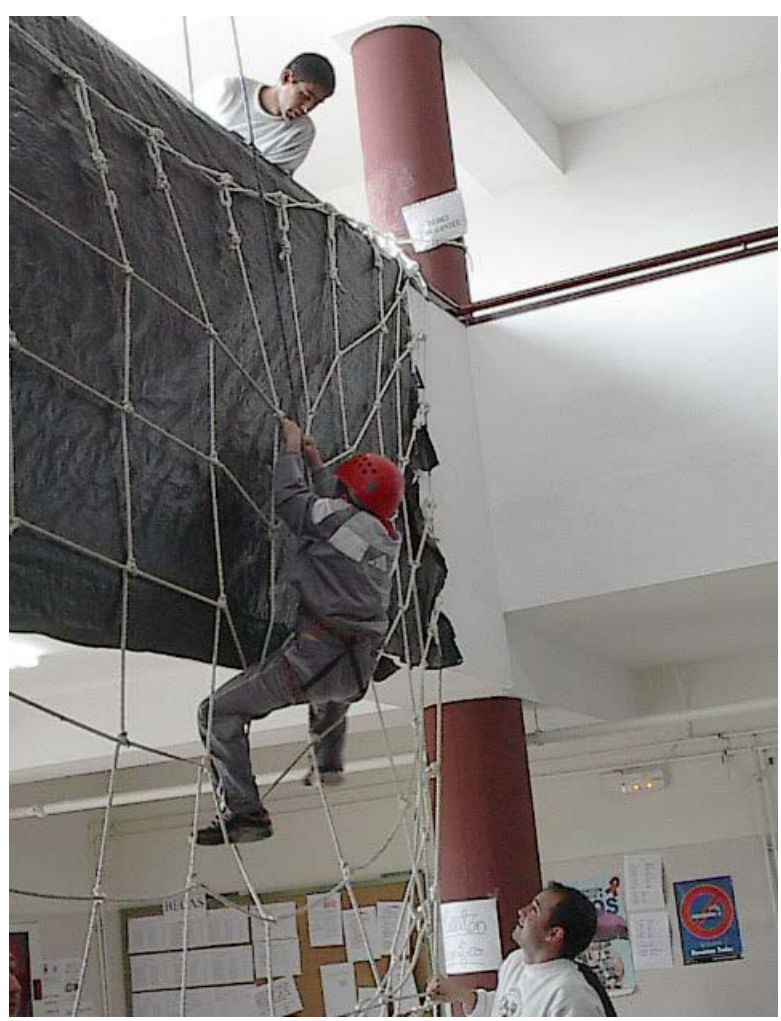


- Delegar responsabilidades e integrar sus aportaciones.

\section{Buscar contactos externos:}

- Asociaciones del entorno (amas de casa, deportivas, juveniles, ...).

- Instituciones públicas: centro de formación del profesorado, área de juventud y deportes del Ayuntamiento y de la Diputación, proyectos en marcha donde se encuadre la actividad, fondos europeos...

- Federaciones deportivas del entorno.

- Colaboración con otros institutos.

- Ofrecer la posibilidad de realizar prácticas a los alumnos de los ciclos formativos de grado medio y superior de educación física, magisterio de educación física y ciencias del deporte.

8. Realización del proyecto definitivo mediante la formación de una comisión:

- Marcar objetivos generales.

- Analizar los recursos humanos, materiales y económicos de los que se disponen.

- Analizar las posibilidades que tiene el centro (estructuras físicas para la creación de espacios de aventura).

- Decidir que actividades se van a realizar en función del $n^{\circ}$ de monitores y material.

- Decidir el número de participantes en función de los monitores disponibles (ratio 1 ) 15 como máximo por monitor/a).

- Definir las tareas a realizar por grupos/ comisiones.
- Conocer las características de los alumnos con necesidades educativas especiales, para adaptar actividades.

- Poner un nombre a la actividad que nos permita difundirla y conseguir subvenciones y apoyos externos.

- Crear un hilo conductor o eje de la actividad mediante la elección de un tema de repercusión social, que tenga un fin educativo.

- Temporalizar las actuaciones.

* La comisión estará formada por los diferentes entes participantes: profesores, alumnos, colaboradores externos, padres y madres,...

9. Primera reunión de todos los interesados, para:

- Comunicar los puntos claves del proyecto.

- Tener en cuenta variaciones o modificaciones a partir de las aportaciones de los organizadores.

- Temporalizar las actuaciones.

- Ajuste del presupuesto.

10. Búsqueda de subvenciones y ayudas:

- Ayudas económicas: convocatorias de la administración educativa correspondiente (Junta de Extremadura,...), ayuntamientos, obras sociales de cajas o bancos, centro de profesores y recursos, AMPA, etc.

- Ayudas materiales y recursos humanos: otros institutos y colegios, asociaciones, federaciones deportivas.

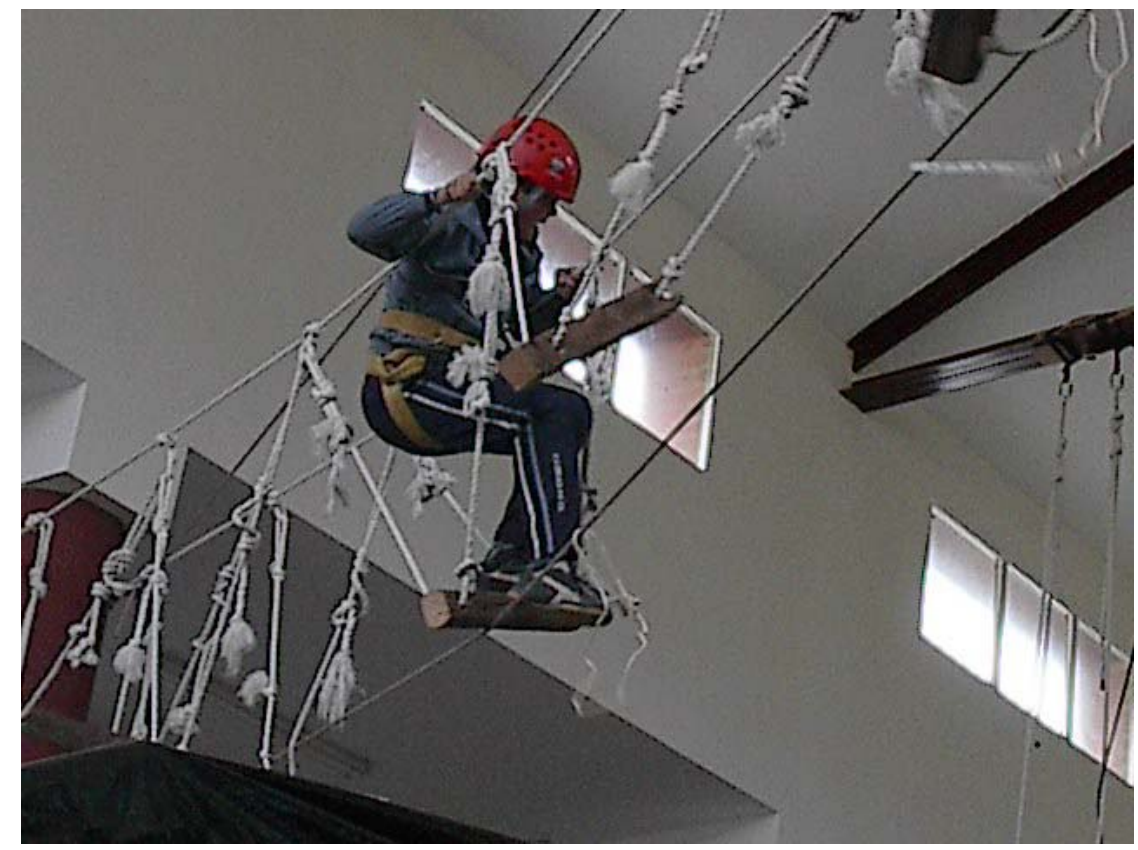




\section{SEGUNDO TIEMPO ORGANIZATIVO.}

(Pronto comienza la actividad, es necesario ultimar todos los detalles)

1. Desarrollar los diferentes talleres y actividades:

- Facilitar la comunicación con el coordinador y entre grupos de trabajo (Internet, móviles, tablón de anuncios).

- Detectar las dificultades y aportar soluciones.

- Definir la ubicación de la actividad.

- Determinar los recursos materiales y humanos necesarios para su puesta en práctica.

- Prever distintas contingencias (la meteorología, forma de dinamizar cada posta, como actuar en caso de emergencia, posibles interferencias con actividades cercanas,...).

2. Publicidad de la actividad:

2.1. En el centro: actuaciones para predisponer a los alumnos y profesores.

- Generar expectación a todos los miembros de la Comunidad Educativa,

- A través de las diferentes asignaturas, se les dará información sesgada a tos alumnos, de las actividades que se van a realizar, involucrando a su vez a los profesores.

- Convocar un concurso de carteles entre los chavales, del que saldrá el de la fiesta.

- Informar sobre la grabación de la fiestay la posterior visualización en diversas clases (o recreos).

- Preparar una actividad final que favorezca las relaciones interpersonales entre todos los participantes en la fiesta (concurso gastronómico, comida conjunta, etc.).

\subsection{Al exterior:}

- Informar a los medios de comunicación de la fiesta, invitándoles a la actividad (notas de prensa, tríptico, informe).

- Mandar invitaciones a los diferentes entes colaboradores (Centro de profesores y recursos, Diputación, Ayuntamiento, Asociaciones, Federaciones, ....).

- Invitar a los centros colindantes.

3. Revisar la cobertura legal de la actividad.

4. Contactar con los diferentes entes públicos: Policía local, Ayuntamientos, Guardia Civil, Bomberos, Delegación de Educación, Centros de salud, etc., y explicarles qué vamos a hacer y en qué pueden colaborar y/o participar: personal humano, medios materiales, infraestructura, etc.

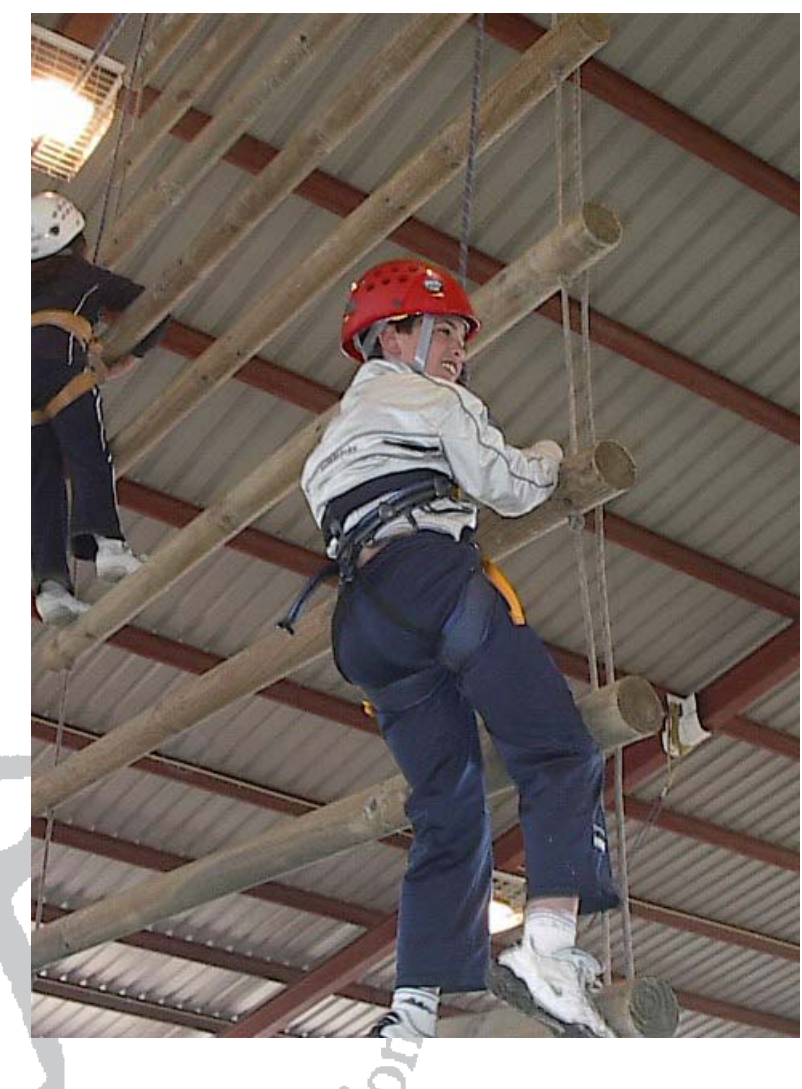

5. Localización y gestión del material:

- Contactar con los diferentes proveedores de material (asociaciones, Federaciones, Institutos, Centros de profesores, empresas, Diputación, Universidad,...).

- Negociación para la consecución del material (fecha del préstamo, inventario y reposición del posible material deteriorado).

- Medio de transporte hasta el centro.

- Almacenamiento del material (debido al gran valor y volumen del mismo).

- Revisar el estado del material.

6. Montaje de la exposición de dibujos y carteles en el centro.

7. Reunión informativa:

- Revisar la temporalización.

- Analizar los imprevistos que han surgido y tomar nota para ediciones próximas.

- Realizar los ajustes necesarios.

- Actualización de cuentas.

- Generar una red de información profesores-alumnos sobre:

- ¿Cómo va a funcionar todo? (hora de comienzo y finalización, rotación en las actividades, etc.).

- Lugares específicos (botiquín, guardarropa, etc.). 


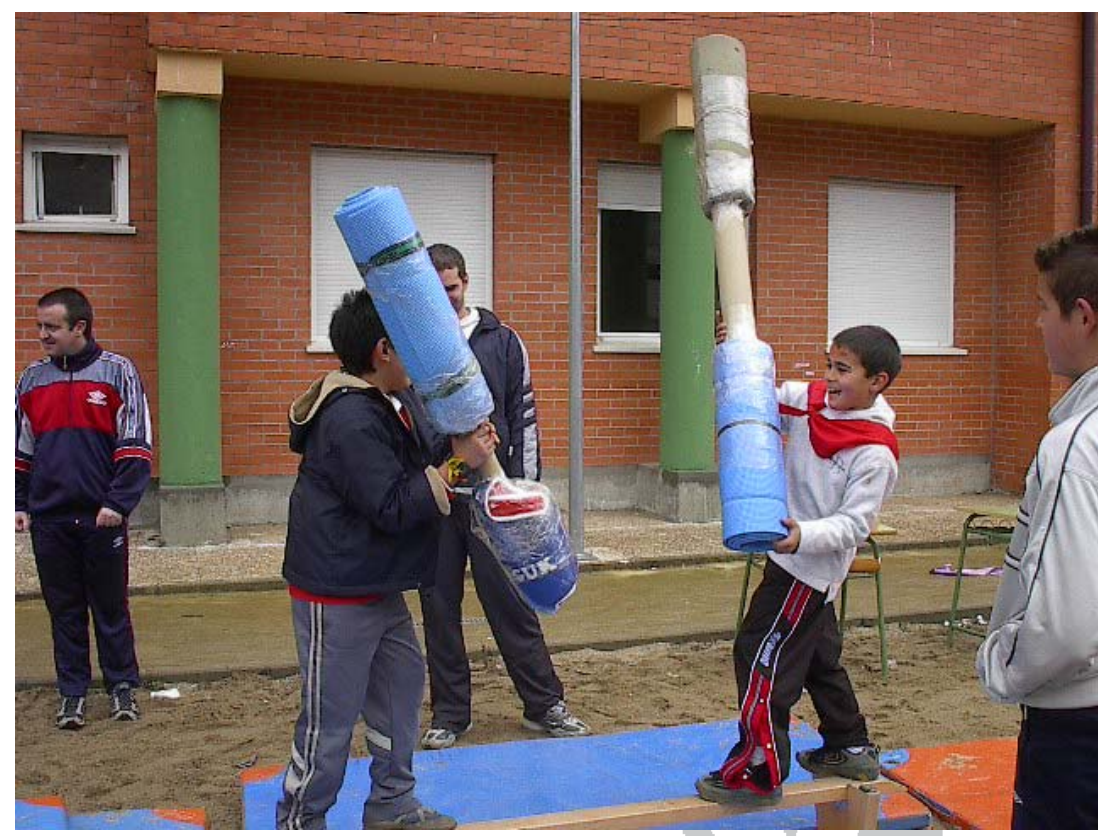

- Ropa que han de traerse los participantes (bañador, toalla, ropa para cambiarse).

- Distribución de los profesores y alumnos colaboradores en las actividades.

- Entornos de práctica (según edad, según destreza, etc.).

- Puntos de encuentro y lugares con acceso no permitido.

- ¿A quién dirigirse en caso de emergencia?

- ¿Cómo y cuándo se recoge el material?

- Horario general.

FASE ACTIVA.

\section{TERCER TIEMPO ORGANIZATIVO}

(Desde que se empieza a montar, hasta que se termina de recoger)

1. Reunir a los diferentes organizadores y colaboradores para informar a pie de campo de:

- Protocolos de actuación dentro de la actividad.

- Protocolos de seguridad.

- Reparto de receptores de corto alcance. (Todos en la misma frecuencia y con batería llena)

- Entrega de mapas con la ubicación de:

- Puntos de encuentro y organización.

- Distribución de actividades según edades y habilidades.

- Almacén de materiales.
- Aseos.

- Primeros auxilios.

2. Organización de los recursos humanos.

3. Repartir el material de cada posta.

4. Prever la toma de imágenes en video y fotografía.

5. Distribuir y montar las actividades. (Control exquisito de la contingencia y del material).

6. Repasar los protocolos de seguridad en los montajes.

7.......... Y. iiiiiiiiiiiiiiiiiiTODOS A

DISFRUTAR!!!!!!!!!!!!!!!!

ELÉXITO DE LA FIESTA

AHORA DEPENDE DE VOSO-

TROS, LA DINAMIZACIÓNY ELENTUSIASMO DELOS MONITORES, COLABORADORES, ORGANIZADORES,...

9. Atención a los medios de comunicación y representantes de las organizaciones y personas relevantes.

10. Desmontar, inventariar y revisar el material.

11. IA REPONER LASENERGÍAS GASTADAS! Disfrute de viandas típicas de la tierra:

* No olvidamos que el momento de la comida es un proceso socializador que favorece la comunicación y reflexión espontánea entre los comensales.

\section{FASE POSTACTIVA}

\section{CUARTO TIEMPO ORGANIZATIVO.}

(Análisis, reflexión y recopilación de lo acontecido)

1. Última reunión de la comisión:

- Reflexión en forma de «lluvia de ideas».

- Evaluación del grado de satisfacción del profesorado.

- Anotación de los fallos detectados y posibles soluciones.

- Cierre de cuentas.

- Elaboración y distribución de certificados a los colaboradores.

2. Puesta en práctica de actividades que fomenten la reflexión de los alumnos des de distintas asignaturas.

* Esta reflexión contribuye al tratamiento de contenidos de forma interdisciplinar.

Análisis de lo acontecido con los diferentes Entes colaboradores y organizadores. 


\section{Relación de posibles actividades}

\section{Aventura}

1.1 Piragüas.

1.2 Rapel.

1.3 Escalada horizontal.

1.4 Escalada en tacos de madera.

1.5 Escalada en cajas.

1.6 Circuito de bicicletas.

1.7 Tirolinas.

1.8 Parapente.

1.9 Circuito de aventura pequeño.

1.10 Plástico deslizante.

1.11 La bala humana.

1.12 Péndulos.

1.13 Botafumeiro humano.

1.14 Redes colgantes.

1.15 Barrancos.

1.16 Contenedor humano.

1.17 Tablas rodantes

1.18 Caballos.

1.19 Tiro con arco.

1.20 Saco de obra.

1.21 Escalera gigante.

1.22 Techo de redes.

1.23 Pantano.

1.24 Pesca.

1.25 Escalas.

1.26 Puentes.

1.27 Ascensión por red.

1.28 Tobogán.

1.29 Escalas.

1.30 Buceo.

1.31 Bicho palo.

1.32 Bosque de los ausentes.

1.33 Rocódromo.

1.34 Escalada en madera.

1.35 Red gigante.

1.36 Orientación - Juego del tesoro.

1.37 Escalada en edificio.

1.38 Escalada a baja altura.

1.39 Troncos colgantes.

1.40 Hamacas gigantes.

1.41 Los murgañinos.

\section{Deportivas}

2.1 Salvamento.

2.2 Boxeo.

2.3 Taekwondo.

2.4 Esgrima.

2.5 Balón Korf.

2.6 Balonmano.

2.7 Voley playa.

2.8 Rugby.

2.9 Fútbol

2.10 Palas.

2.11 Bádminton.

2.12 Indiacas.

2.13 Cama elástica.

2.14 Hockey.

2.15 Taichi.

2.16 Capoeira.

2.17 Lacrosse.

2.18 Críquet.

2.19 Mini voleibol.

2.20 Mini balonmano

2.21 Baseball.

2.22 Mini tenis de mesa

2.23 Fútbol Tenis.

2.24 Aerobitrón.

2.25 Defensa personal.

\section{Recreativas}

3.1 Ultimate.

3.2 Cometas.

3.3 Gimnasia colectiva.

3.4 Juegos populares.

3.5 Zancos.

3.6 Futbolín humano.

3.7 Balones gigantes.

3.8 Malabares.

3.9 Paracaídas.

3.10 Discos voladores.

3.16 Monopatín.

3.17 Zancos.

3.18 Juegos populares.

3.19 Gimnasia colectiva (acrosport)

3.20 Boomerang.

$3.21 \mathrm{Gol}$ y gol.

3.22 Balón asesino. 
3.23 Comba.

3.24 Chito-Chito.

3.25 Bolos.

3.26 Cucaña.

3.27 Gusanos rodantes.

3.28 Gladiadores.

3.29 Pistola de tenis.

3.30 Bolos humanos.

3.31 Tan gran gigante.

3.32 Actividades de confianza.

3.33 Juegos de reto.

3.34 Juegos de resolución problemas.

\section{Bibliografía}

Alonso, V. M. (1992). La evolución del área de actividades en la naturaleza, Alminar, 23, 1416.

Cuenca, M. (1995). La alternativa del juego I y II. Juegos y dinámicas de educación para la paz. Madrid: Los libros de la catarata.

Farias, E. y Torrebadella, X. (1995) Hacia una metodología de análisis de los espacios deportivos naturales susceptibles a la práctica de actividades físicas de aventura en la naturaleza. Parque Nacional de Aigües Tortes y Lago San Maurice. Apunts: Educación física y deportes, 41, 137-140. Barcelona: Generalitat de Catalunya

\subsection{Boccia.}

4.2 Juegos sensibilizadores.

4.3 Circuito en silla de ruedas.

4.4 E.F. Adultos.

4.5 Fútbol para ciegos (goal ball).

\section{Animación}

5.1 Túnel de las sensaciones.

5.2 Pasaje del terror.

5.3 Gran Juego de la oca.

5.4 Twister gigante.

5.5 Tartazo.

5.6 Piscina de espuma.

5.7 Pasacalles.

5.8 Danza contemporánea.

5.9 Bailes de salón.

5.10 Danzas del mundo.

5.11 Cuenta cuentos.

5.12 Maquillaje.

\section{Otras actividades}

6.1 Ecobús.

6.2 Repoblación piscícola de truchas.

6.3 Don Limpio.

6.4 Reforestación.

6.5 Taller de huellas de animales.

6.6 Exposición de flora de Extremadura.

6.7 Exposición de espacios naturales de Extremadura.

6.8 Red natura 2000.
Guillén, R. y Lapetra, S. (1995) Aproximación metodológica a las actividades de enseñanzal aprendizaje en esquí: un modelo integral de actuación. II Congrés de les ciéncies de l'esport, I'educació física i la recreació de L' INEFCLleida. Ámbits especifics dels esports il' educació física. II Vol. Pp. 353-364. Lleida. Generalitat de Catalunya.

Gutiérrez Sanmartín, M. y González de Martos, M. (1995) Deportes de riesgo y aventura: una perspectiva psicosocial del paracaidismo. Revista Española de Educación Física y Deportes. Vol. 2 No 3.Pp. 30-39.

Parra, M. y Rovira, C. (2002). Jugando con fuego. Propuestas pedagógicas al calor de las sensaciones de aventura, Tándem, 6, 51-64.

Parra, M. y col. (2000). Valores educativos de la aventura interior.

I Congreso nacional de Deportes en Edad Escolar. Sevilla: Excmo. Ayuntamiento de Dos Hermanas. Patronato Municipal de Deportes.

Ziegenspeck, J. (1996). La pedagogía de la experiencia: ejemplo del instituto de Alemania, Apuntes del curso de especialización en ocio y recreación deportiva. Facultad de Ciencias del Deporte de Cáceres. Paper. 